\title{
Listening to the Appeal from the Wild
}

\author{
Christophe Boesch \\ Department of Primatology, Max Planck Institute for Evolutionary Anthropology, Leipzig, Germany \\ Corresponding author (Email: boesch@eva.mpg.de) \\ Citation - Boesch, C. (2020). Listening to the appeal from the wild. Animal Behavior and Cognition, 7(2), $257-263$. \\ doi: https://doi.org/10.26451/abc.07.02.15.2020

\begin{abstract}
Fashion in science is like in other domains, it is strongly influenced by what we would call nowadays "influencers" that set the scene to start a new trend. In this sense, the "Vervet Monkey Alarm Calls" paper was an "influencer" and gave to many of the younger generation the strength to embark in the study of animal cognition in the wild. The very precise experimental procedures adopted in the paper, eliminating step-by-step the alternatives and finishing by proposing fascinating new insights into the "Monkey Mind" has enthralled cohorts of researchers and provided strong support for two propositions: First, you can do cognitive research on wild animals, and second, monkeys are smarter than you think! However, influencers are not always strong enough to change the field and, in my opinion, animal cognition research would greatly profit from a renewed interest in the approach pioneered by the "Vervet Monkey Alarm Call."
\end{abstract}

Keywords - Cognition, Field work, Captive experiments, Ontogeny, Environment, Sociality

\section{Cognitive Research with Wild Animals is Possible}

The field of animal cognition has long had a strong fondness for strictly controlled experiments performed in the lab on captive individuals. The main justification being that too many factors could affect the behavior of an individual in the wild, so that one needs captive conditions to allow for strict control of such confounding variables. In addition, and understandably, it is more comfortable for scientists to work in the lab than in a remote place in Africa with bugs, potentially dangerous predators, and strenuous climatic conditions. Taking the opposite stance, Seyfarth, Cheney and Marler (1980) summarized a long series of experiments done in the wild, refuting the imperative that only captive experiments are valid in cognitive science. They were successful at showing intriguing new aspects of monkey cognition in which they revealed naturally semantic communication.

To many psychologists, experiments are the golden standard without which no conclusion can be drawn about cognition. To that end, animals are kept in captive conditions that allow for better control about the prior knowledge individuals acquire before being used in an experiment at the cost of having the animals living in much poorer social and ecological environments than in nature. To some, the ontogenic validity of such living conditions might be too disruptive to allow for normal cognitive development (Birkett \& Newton-Fisher, 2011; Brüne et al., 2006), and certainly limits extremely the prior-knowledge the individual might acquire about life (Frith, 2007). Then, in captive experiments, the individual is routinely presented with conditions in which they have to press at bars or touch computer screens to obtain food, to turn cups to track the location of different objects, to pull at ropes or rake with sticks to reach food outside the cage (see reviews in Povinelli, 2000; Tomasello \& Call, 1997). Experimenters seem to assume that, notwithstanding the artificiality of the situation, the tested animals 
will be highly motivated to find out about the meaning of the task presented and to follow the human experimenter's logic. Do we reasonably expect animals to have enough of a "human" mind to understand the logic of such experiments? Would it not be more sensible to concentrate on cognitive abilities that present some direct resemblance with the contexts under which their cognition evolved? Sure, the tested individuals have been laboriously conditioned through many hundreds of pre-testing tasks to learn to use those novel objects, but is cognition only about conditioning?

The limited ontogenic and ecological validity of such experiments questions the scientific value of these results, and would at least require special scrutiny when a cognitive limitation is suggested for the whole of the species from such artificial experiments (Benson-Amram et al., 2013; Boesch, 2007; 2012; Calisi \& Bentley, 2009; Marino \& Frohoff, 2011; Suddendorf \& Whiten 2001). While the question of ecological validity has been addressed by some authors in the past (Boesch, 2007, 2012; Calisi \& Bentley, 2009; Cauchoix et al., 2017; Haun \& Tomasello, 2016; Lambrechts et al., 1999; Marino \& Frohoff, 2011; Mason, 2010; Morand-Ferron et al., 2015; 2019; Pack, 2010; Stevens \& Carlson, 2008; Suddendorf \& Whiten, 2001), some authors proposed that the difference between captive and wild animals are so important to the point of asking if they are the same animals (Calisi \& Bentley, 2009; Marino \& Frohoff, 2011), while others proposed that a synergy between captive and naturalistic approaches would be the ideal solution to gain a full understanding of an animal cognitive abilities (Pack, 2010; Tomasello \& Call, 2011). Some contradicting results from captive bird studies with the closely related species did make sense only after a precise consideration of the different ecologies of these species (Healy et al., 2009; Lambrechts et al., 1999; Pravosudov \& Smulders, 2010; Smulders et al., 2010). Furthermore, the assumption that all species should react in a same way to captivity made by comparative psychologists has been tested thanks to a well-developed body of research on animal welfare, stressing that even closely related animal species may differ extensively in their reactions to captivity (Mason, 2010). A comparison of twelve primate species revealed that small arboreal species are particularly threatened by the presence of humans (Chamove et al., 1988) ${ }^{1}$. Wide-ranging primate species living naturally in large social groups present more hair-pulling behavior and stereotypic pacing levels than smaller body-sized ranging ones in captive conditions (Pomerantz et al., 2013, see Clubb \& Mason, 2003 for a similar effect in carnivores). Partly due to these issues, an increasing number of researchers have followed the tracks of Seyfarth and colleagues and performed well-controlled experiments in the wild on wild living individuals (Cauchoix et al., 2017; van de Waal et al., 2010; Flombaum \& Santos, 2005; Santos et al., 2006; St Clair \& Rutz, 2013).

At the same time, neuroscientists have provided vast new information on the effects of impoverished social and physical environments on the development of the brain and cognition that should force scientists to reassess their attitude towards captive animal studies. While in the 80s, it was still believed that, once matured, the brain remains rigid with limited regeneration potential, if at all (reviewed in Hillman et al., 2008; Kolb et al., 2017; Praag, 2009), starting around the 1990s, numerous studies have accumulated documenting the plasticity of the brain that remains for the life of the individuals. Looking more precisely into brain plasticity, it became rapidly evident that the development of the brain structures is negatively affected when the individual develops in a poor social and physical environment (Hänggi et al., 2010, 2014, 2015; Maguire et al., 2000; Praag et al., 2000). These detrimental effects have now been convincingly documented in humans, in different primate species, in rodents as well as in fishes and birds. Furthermore, this plasticity is observed not only in the developing brain, but also in the adult brain sometimes showing less or more plasticity than in the juvenile brain. The most striking and upsetting example came from Romanian orphanages in which living conditions were such that the orphans presented dramatic delays in physical growth and cognitive development (e.g., Nelson et al., 2007; Smyke et al., 2010). However, equally strong negative effects have been documented in the development of the brains of chimpanzees when not reared by their mothers (Bogart et al., 2014). This effect was so strong that it was still visible in individuals in their 40s. Similarly, adult marmosets present much higher

\footnotetext{
${ }^{1}$ Among them are vervet monkeys that were studied by Seyfarth, Cheney, and Mason (1980): due to their small size, the presence of humans in captivity was resented as quite stressful compared to the larger baboons.
} 
dendritic spine density and synapse number in the hippocampus and the pre-frontal cortex even after a brief stay in a moderately complex environment (Kozorovitskiy et al., 2005). Since those two areas of the brain are known as important for cognition and are involved in foraging and navigating in complex environments, these results represent a serious concern about placing animals in even slightly deprived captive conditions. In many of the studies about brain plasticity, the scientists measured cognitive performance and could show that important cognitive abilities such as learning, memory, spatial knowledge, executive functions, and causal reasoning are dramatically improved the more physical exercise and the more complex the social and physical environment the individuals have experienced (e.g., Hillmann et al., 2008; Piepmeier et al., 2015). In other words, nowadays it is not possible to ignore the detrimental consequences on the brain and on cognition of an individual growing under even slightly impoverished conditions ${ }^{2}$.

The full breadth of brain plasticity still needs to be fully understood, but some are already suggesting that "at least $80 \%$ of the brain grey matter is modifiable by physical activities" (Batouli \& Saba, 2017, p. 204, but see Esteban-Cornejo et al., 2018). Moreover, we need to take into account the pleiotropic functioning of neuronal cells within networks in the brain (Anderson, 2010; Anderson et al. 2013). For example, on average, 78 anatomical regions of the brain were active in 95 tasks spread across nine cognitive domains (Anderson \& Penner-Wilger, 2013): "The upshot: local neural circuits are not highly selective, and typically contribute to multiple tasks across domain boundaries" (p. 44). Taken together, this illustrates the potential large positive impacts of growing up in a rich social and ecological environment, as potentially each region of the brain is participating in many different cognitive tasks. A small detrimental effect in one brain region will be amplified by this plastic participation in many different cognitive activities.

Supporters of captive studies have countered with the argument that captive studies revealed so many impressive cognitive abilities, like about theory of mind or metacognition, that their contribution to cognition cannot be denied (e.g., Tomasello \& Call, 2008). This argument is beside the point, as the real question is how much more or different would the cognitive achievements of different animal species be if the living conditions they experienced during their ontogeny and during the experiments were taken into account. As scientists, it should be our duty to make sure that we are not introducing a systematic bias in our approach and I feel that all the problems with captivity mentioned above need to be addressed. After decades of comparative psychology, for the first time, a project on brain connectivity will compare the structure of wild and captive chimpanzee brains, of individuals whose whole life history is known (see Catherine Crockford - www.eva.mpg.de/ecology/projects-and-research-groups/evolution-of-brainconnectivity). This will allow the first test of the prediction that wild brains are more developed in functional areas important for their ecological and social needs (Boesch, 2007, 2012).

By studying the vervet monkeys in their natural social and ecological environment, Seyfarth, Cheney and Marler (1980) addressed directly the issue of ontogenic and ecological validity of those captive experiments. To do so, they moved out to some remote places in Africa, to the natural habitat of the vervet monkeys, and they adapted their experimental equipment and procedures to the socioecological conditions prevailing in Kenya. They concentrated their experiments on one vital aspect of their life; communication about life-threatening predators. Such situations are known to be crucial for their survival and were therefore most likely to be under strong selective pressure. This new paradigm allowed them to uncover so far unknown abilities in vervet monkeys that would still be ignored if limited to captive studies. Vervet monkeys' alarm calls were tuned to the type of predators they face. The success of their method in uncovering new aspects of the vervet monkeys' mind encouraged them to address successfully many new issues of social cognition, and they published those in a book entitled "How Monkeys See the World" in 1990.

\footnotetext{
2 Typically, environment enrichment by adding just some tubes, balls and a running wheel in a naked cage suffice to elicit dramatic changes in the brain structures in a group of rats (Praag et al., 2000). We are therefore still a far cry away from any environment enrichment similar to the challenges that a wild environment would pose to an individual.
} 
The success of their approach and the discovery of new facets of the vervet monkeys' mind gave many young scientists the courage to follow their steps and work on many different animal species to reveal unknown and partly unexpected facets of the primate mind. As the Seyfarths anticipated, comparative psychology needs to consider the living conditions during ontogeny when studying cognition to progress in the understanding of species-specific abilities and cognitive evolution. This implies that one needs to consider population differences in cognition within the same species, including in chimpanzees and humans (Boesch, 2007; Henrich et al., 2010). This makes comparison more complex, but at the same time, all the more fascinating.

\section{Animals Can Achieve More than you Think}

Humans view themselves as the crown of evolution and tend to look down with some condescendence at monkeys and chimpanzees. Consequently, not much thought is given when accepting results confirming the human pre-eminence, while any results threatening humans' outstanding position are carefully and critically scrutinized. Bertrand Russell (1918) proposed that this human attitude was at the origin of many myths in science. In their work, Seyfarth and colleagues made it clear that vervet monkeys in the wild can distinguish different predators, and, through their vocalization, communicate to group members the presence of distinct predator types to which they respond accordingly. These results substantiate a general trend; i.e., the more we study a species in detail, the more cognitively evolved it appears.

In addition, studying animals in their natural environment is the best way to realize the precise evolutionary challenges they have to face that select for cognitive abilities. The rainforest of the Taï National Park, Côte d'Ivoire, is inhabited by 11 different species of monkeys that forage for food high up in the canopy. When I see them jumping between branches $50 \mathrm{~m}$ high above the ground, chasing one another in an impressive aerial circus, it seems to me they are taking high risks of killing themselves if falling to the ground. However, in 40 years of work in African forest, I never saw a monkey jump inadvertently on a branch not large enough for its weight, not attached to its support, or too far away for his jumping ability. Obviously, to survive in such an environment, they developed some distinctive abilities to evaluate the physical properties of branches $50 \mathrm{~m}$ above the ground. Intriguingly, this is in clear contradiction with some conclusions drawn from studies about the knowledge of functional objects, where it was proposed that captive chimpanzees had no knowledge of the invisible properties of objects and of the functional relations between objects (Povinelli, 2000). The captive chimpanzees could not distinguish whether a tool was functionally connected or not to a piece of food, or whether a rubber tool was suitable to move the food! In the safe, predictable and limited environment of captivity, individuals do not need to predict about object resistance, stability or connectivity, and so it should not come as a surprise that they have limited abilities to do so. However, judging wrongly the physical properties of branches high up in trees is a matter of life and death. It remains very hard for a ground-dwelling human to imagine what it takes to move high up in trees. I experienced this a few times when building platforms high up in these trees, and to my shame, was too scared to walk on such branches, let alone stand on the platform!

As I studied the nut-cracking behavior of wild chimpanzees, I had to evaluate their abilities to select proper hammers to crack the nuts at trees with no hammer available. The Taï forest is very dense so that visibility on the ground rarely exceeds $30 \mathrm{~m}$; thus, finding a stone heavy enough to open a hard nut is a challenge, as big stones are rare in this forest. To understand how chimpanzees could find those stones, and what criteria they used when selecting one, I marked all the stones large enough to crack a hard Panda nut, and recorded their precise location. By doing so, I realized that, on average, chimpanzees choose among five stones within a circle of $300 \mathrm{~m}$. In addition, this selection was not random, as they chose the closest stones to the goal tree in $63 \%$ of the transports and the one requiring the least transport energy in $53 \%$ of the transports (Boesch \& Boesch, 1984). This ability to compare distances oriented in different directions in space takes up to nine years in children, as long as they can see all the objects (Piaget \& Inhelder, 1947). Would humans achieve this, if the distances between objects were not visible? I am not 
aware of any study exploring this in humans. The point is that some of these stones weigh more than 4 to $5 \mathrm{~kg}$ and carrying them over many hundreds of meters without a precise notion of straight lines and direction would be very costly, and nut-cracking would not be energetically worthwhile. So, the selective pressures to acquire such skills are important in the dense forest. In addition, such skills are also beneficial to find large but rare fruit trees in the forest that produce the juicy fruits chimpanzees feed on most of the year (Janmaat et al., 2013; Normand \& Boesch, 2009). These trees are often up to 2,000 m apart and finding them without a precise knowledge of their locations would be very time consuming and energetically costly (Normand et al., 2009). So, the selection pressure is going to be high on forest chimpanzees to acquire the necessary spatial skills when living in such a low visibility environment. All I can say is that after walking for 10 years in Taï forest, I was still not able to walk in a straight line between two of these trees.

All these cognitive abilities about object functionality, spatial knowledge, causality understanding cannot be studied in captivity in the natural scale important to the survival of the individuals, and therefore, we are bound to underestimate the cognition of a species if we study it only in captivity. Seyfarth, Cheney, and Marler (1980) warned us about this aspect in their paper, as they explained that cognitive skill essential for survival of the individuals are going to be selected for in the individuals living there. In other words, if one wants to understand the cognitive development of given species, one needs to study them in all the different types of environment and challenges they face. In their more recent book "Baboon Metaphysics, The Evolution of a Social Mind", Cheney and Seyfarth (2007) were even more explicit about the importance of the ecological challenges faced by the individuals in shaping their cognitive abilities. It seems obvious that in a population where $53 \%$ of the infant mortality is due to infanticide, and where $95 \%$ of the adult females are killed by predators (Cheney \& Seyfarth, 2007), the selective pressures on cognition are completely different than in a safe permanently food-provisioned captive environment. So, it should not come as a surprise that animals can be smarter than expected under captive conditions. This essential lesson needs still to be acknowledged by many in comparative science to not systematically underestimate the cognitive skills of different animal species. I am not claiming captive studies are useless for studying cognition; first, they have for long been the only sources of our knowledge on animals before field studies were started, and second, many aspects of the morphology, physiology and some behavior patterns of animals are less influenced by the ontogenic and ecological factors than cognition. What I am saying is that the increasing recent awareness about the negative effects of living in deprived social and ecological conditions should force scientists to reevaluate the value of such captive research.

\section{Acknowledgements}

After 40 years of research, I realized that I owe the most to the wild chimpanzees of the Tai National Park, in Côte d'Ivoire, that accepted our presence daily for many years and taught us patiently how to walk in the forest, how to follow chimpanzees and what it means to be a chimpanzee in a dense African rainforest. With time, I appreciate more and more what unique privilege this has been and how uniquely important their lessons were to understand the uniqueness of chimpanzees and humans. After 40 years, I am still learning from them and I thank them wholeheartedly for this.

\section{References}

Anderson, M. (2010). Neural reuse: A fundamental organizational principle of the brain. Behavioral and Brain Sciences, 33, 245-313.

Anderson, M., Kinnison, J., \& Pessoa, L. (2013). Describing functional diversity of brain regions and brain networks. NeuroImage, 73, 50-58.

Anderson, M., \& Penner-Wilger, M. (2013). Neural reuse in the evolution and development of the brain: Evidence for developmental homology? Developmental Psychobiology, 55, 42-51.

Batouli, S., \& Saba, V. (2017). At least eighty percent of brain grey matter is modifiable by physical activity: A review study. Behavioural Brain Research, 332, 204-217. 
Benson-Amram, S., Weldele, M., \& Holekamp, K. (2013). A comparison of innovative problem-solving abilities between wild and captive spotted hyaenas, Crocuta crocuta. Animal Behaviour, 85, 349356.

Birkett, L., \& Newton-Fisher, N. (2011). How abnormal is the behavior of captive, zoo-living chimpanzees? PLoS One, 6, e20101.

Boesch, C. (2007). What makes us human (Homo sapiens)? The challenge of cognitive cross-species comparison. Journal of Comparative Psychology, 121, 227-240.

Boesch, C. (2012). Wild cultures: A comparison between chimpanzee and human cultures. Cambridge University Press.

Boesch, C., \& Boesch, H. (1984). Mental map in wild chimpanzees: An analysis of hammer transports for nut cracking. Primates, 25, 160-170.

Bogart, S., Bennett, A., Schapiro, S., Reamer, L., \& Hopkins W. (2014). Different early rearing experiences have long-term effects on cortical organization in captive chimpanzees (Pan troglodytes). Developmental Science, 17, 161-174.

Brüne, M., Bruen-Cohrs, U., McGrew, W., \& Preuschoft, S. (2006). Psychopathology in great apes: Concepts, treatment options and possible homologies to human psychiatric disorders. Neuroscience and Biobehavioral Reviews, 30, 1246-1259.

Calisi, R., \& Bentley, G. (2009). Lab and field experiments: Are they the same animal? Hormones and Behavior, 56, $11-10$.

Cauchoix, M., Hermer, E., Chaine, A., \& Morand-Ferron, J. (2017). Cognition in the field: Comparison of reversal learning performance in captive and wild passerines. Scientific Reports, 7, 12945.

Chamove, A., Hosey, G., \& Schaetzel, P. (1988). Visitors excite primates in zoos. Zoo Biology, 7, 359-369.

Cheney, D., \& Seyfarth, R. (2007). Baboon metaphysics: The evolution of a social mind. The University of Chicago Press.

Clubb, R., \& Mason, G. (2003). Captivity effects on wide-ranging carnivores. Nature, 425, 473-474.

Esteban-Cornejo, I., Catena, A., Hillman, C., Kramer, A., Erickson, K., \& Ortega, F. (2018). Commentary: At least eighty percent of brain grey matter is modifiable by physical activity: A review study. Frontiers in Human Neuroscience, 12, 195.

Flombaum, J., \& Santos, L. (2005). Rhesus monkeys attribute perceptions to others. Current Biology, 15, 447-452.

Frith, C. (2007). Making up the mind: How the brain creates our mental world. Blackwell Publishing.

Haun, D., \& Tomasello, M. (2016). How to compare across species. Psychological Science, 27, 1670-1672.

Healy, S., Bacon, I., Haggis, O., Harris, A., \& Kelley, L. (2009). Explanations for variation in cognitive ability: Behavioural ecology meets comparative cognition. Behavioural Processes, 80, 288-294.

Henrich, J., Heine, S., \& Norenzayan, A. (2010). The weirdest people in the world? Behavioral and Brain Sciences, $33,61-135$.

Hillman, C., Erickson, K., \& Kramer, A. (2008). Be smart, exercise your heart: Exercise effects on brain and cognition. Nature, 9, 58-65.

Janmaat, K., Ban, S., \& Boesch, C. (2013). Chimpanzees use long-term spatial memory to monitor large fruit trees and remember feeding experiences across seasons. Animal Behaviour, 86, 1183-1205.

Kolb, B., Harker, A., \& Gibb, R. (2017). Principles of plasticity in the developing brain. Developmental Medicine \& Child Neurology, 59, 1218-1223

Kozorovitskiy, Y., Gross, C., Kopil, C., Battaglia, L., McBreen, M., Stranahan, A., \& Gould, E. (2005). Experience induces structural and biochemical changes in the adult primate brain. Proceeding of the National Academy of Science, 102, 17478-17482.

Lambrechts, M., Perret, P., Maistre, M., \& Blondel, J. (1999). Do experiments with captive non-domesticated animals make sense without population field studies? A case study with blue tits' breeding time. Proceeding of the Royal Society London B, 266, 1311-1315.

Maguire, E., Gadian, D., Johnsrude, I., Good, C., Ashburner, J., Frackowiak, R., \& Frith, C. (2000). Navigationrelated structural change in the hippocampi of taxi drivers. Proceeding of the National Academy of Science, 97, 4398-4403.

Marino, L., \& Frohoff, T. (2011). Towards a new paradigm of non-captive research on Cetacean cognition. PLoS One, 6, e24121.

Mason, G. (2010). Species differences in responses to captivity: Stress, welfare and the comparative method. Trends in Ecology and Evolution, 25, 713-721.

Morand-Ferron, J., Cole, E., \& Quinn, J. (2015). Studying the evolutionary ecology of cognition in the wild: A review of practical and conceptual challenges. Biological Reviews, 91, 367-389. 
Morand-Ferron, J., Hermer, E., Jones, T., \& Thompson, M. (2019). Environmental variability, the value of information, and learning in winter residents. Animal Behaviour, 147, 137-145.

Nelson, C., Zeanah, C., Fox, N., Marshall, P., Smyke, A., \& Guthrie, D. (2007). Cognitive recovery in socially deprived young children: The Bucharest early intervention project. Science, 318, 1937-1940.

Normand, E., \& Boesch, C. (2009). Sophisticated Euclidean maps in forest chimpanzees. Animal Behaviour, 77, 1195-1201.

Normand, E., Ban, S., \& Boesch, C. (2009). Forest chimpanzees (Pan troglodytes verus) remember the location of numerous fruit trees. Animal Cognition, 12, 797-807.

Pack, A. (2010). The synergy of laboratory and field studies of dolphin behavior and cognition. International Journal of Comparative Psychology, 23, 538-565.

Piaget, J., \& Inhelder, B. (1947). La Représentation de l'Espace chez l'Enfant. Presses Universitaires de France.

Piepmeier, A., Shih, C., Whedon, M., Williams, L., Davis, M., Henning, D., Park, S., Calkins, S., \& Etnier, J. (2015). The effect of acute exercise on cognitive performance in children with and without ADHD. Journal of Sport and Health Science, 4, 97-104.

Pomerantz, O., Meiri, S., \& Terkel, J. (2013). Socio-ecological factors correlate with levels of stereotypic behavior in zoo-housed primates. Behavioural Processes, 98, 5-91.

Povinelli, D. (2000). Folk physics for apes: The chimpanzee's theory of how the world works. Oxford University Press.

Praag, van H. (2009). Exercise and the brain: Something to chew on. Trends in Neurosciences, 32, $283-290$.

Praag, van H., Kempermann, G., \& Gage, F. (2000). Neural consequences of environmental enrichment. Nature Neuroscience, 1, 191-198.

Pravosudov, V., \& Smulders, T. (2010). Integrating ecology, psychology and neurobiology within a food-hoarding paradigm. Philosophical transactions of the Royal Society B, 365, 859-867.

Russell, B. (1918). Proposed Roads to Freedom. Echo Library.

Santos, L., Nissen. A., \& Ferrugia, J. (2006). Rhesus monkeys, Macaca mulatta, know what others can and cannot hear. Animal Behaviour, 71, 1175-1181.

Seyfarth, R., Cheney, D. \& Marler, P. (1980). Vervet monkey alarm calls: Semantic communication in a freeranging primate. Animal Behaviour, 28, 1070-1094.

Smulders, T., Gould, K., \& Leaver, L. (2010). Using ecology to guide the study of cognitive and neural mechanisms of different aspects of spatial memory in food-hoarding animals. Philosophical transactions of the Royal Society B, 365, 883-900.

Smyke, A., Zeanah, C., Fox, N., Nelson, C., \& Guthrie, D. (2010). Placement in foster care enhances quality of attachment among young institutionalized children. Child Development, 81, 212-223.

St Clair, J., \& Rutz, C. (2013). New Caledonian crows attend to multiple functional properties of complex tools, Philosophical Transactions of the Royal Society B, 368, 20120415.

Stevens, N., \& Carlson, K. (2008). Bridging gaps between experimental and naturalistic approaches in the study of primate behavior. International Journal of Primatology, 29, 1395-1399.

Suddendorf, T., \& Whiten, A. (2001). Mental evolution and development: Evidence for secondary representation in children, great apes, and other animals. Psychological Bulletin, 127, 629-650.

Tomasello, M., \& Call, J. (1997). Primate cognition. Oxford University Press.

Tomasello, M., \& Call, J. (2011). Methodological challenges in the study of primate cognition. Science, 334, 12271228.

van de Waal, E., Renevey, N., Favre, C., \& Bshary, R. (2010). Selective attention to philopatric models causes directed social learning in wild vervet monkeys. Proceeding of the Royal Society B, 277, 2105-2111. 